\title{
Equivalent-Circuit Modeling of Nonradiative Surface Plasmon Energy Transfer Along the Metallic Nanowire
}

\author{
Kyungjun Song and Pinaki Mazumder, Fellow, IEEE
}

\begin{abstract}
An analytical methodology for establishing an equivalent-circuit network of nonradiative surface plasmon (SP) energy transport along the metallic nanowire (MNW) is presented. To find out the passive elements for MNW, the SP dispersion and damping relation through modified Bessel function electromagnetic (EM) field expansion was derived, thus demonstrating the low-pas transmission-line (TL) model. Specifically, the lowpass TL parameters, such as series impedance $(Z)$ and shunt admittance $(Y)$ can be calculated based on the lumped-element model and harmonic-voltage (current) distribution. Furthermore, the equivalent-circuit parameters, such as resistance $(R)$, inductance $(L)$, capacitance $(C)$ and conductance $(G)$, are obtained by employing the finite difference (FD) discretization method such as T-cell RLCG networks. These equivalent-circuit elements can be verified by the HSPICE circuit simulation and 3-D scattered finite-difference time-domain (FDTD) method. Finally, the parallel MNWs are modeled as equivalent-circuit networks by using the electrostatic coupling.
\end{abstract}

Index Terms-Finite-difference time-domain method (FDTD), low-pass transmission line (TL), metallic nanowire (MNW), mutual capacitance, surface plasmon (SP), T-cell discretization.

\section{INTRODUCTION}

$\mathbf{T}$ HE miniaturization of optical circuitry in nanoscale dimensions has been aggressively pursued recently with a view to performing on-chip signal and information processing by directly inputting optical data stream from a fiber optics cable to a very large-scale integration (VLSI) chip consisting of billions of nanoscale electronic circuit components [1]-[3]. As a fascinating method, the surface plasmon (SP) that emanates as near-field electromagnetic (EM) wave due to electron oscillation on the interfacing surface between conductor and dielectric material is of great interest for new approaches to obtain the subwavelength waveguide [4]. For example, for a new class of subwavelength photonic elements, there has been much interested in 1-D SP propagation in the metallic structure. By tailoring the topology of a metallic surface, 1-D SP waves can

Manuscript received September 2, 2008; revised May 22, 2009. Date of publication September 29, 2009; date of current version January 26, 2011. This work was supported in part by the Air Force Office of Scientific Research under Grant FA9550-06-1-0493. The review of this paper was arranged by Associate Editor E. Towe.

The authors are with the University of Michigan, Ann Arbor, MI 48109 USA (e-mail:songk@umich.edu; mazum@eecs.umich.edu).

Color versions of one or more of the figures in this paper are available online at http://ieeexplore.ieee.org.

Digital Object Identifier 10.1109/TNANO.2009.2033114 be generated at the metallic surface. It is well known that 1-D SP waves can be produced by the metallic nanoparticles (MNPs) array [4], [5] and metallic nanowire (MNW) [6]-[9]. Especially, the SP in the MNW merits a special attention due to its potential applications in the photonic circuitry [10], subwavelength optical imaging [11], and quantum optics [12].

However, the issue of propagation loss along the MNW remains to be tackled since the dimension contraction, such as transformation from 2-D waves to 1-D waves, increases damping coefficients because low-dimensional waves lead to strong momentum wave. Moreover, this significant energy attenuation in the nanoscale domain leads to substantial difficulties in harnessing plasmonics in on-chip nanoscale waveguide and other near-field optical applications. Furthermore, to address the SP energy dissipation, it is needed to tune the guiding design parameters such as metal physical properties, host matrix physical properties, metal geometries, and operational signal frequency. First, in the case of metal, it would be desirable to choose the noble metals, such as $\mathrm{Ag}$ and $\mathrm{Au}$, having high bulk plasma frequency $\left(\omega_{p}\right)$ and low relaxation frequency $(\gamma)$ to increase the localized EM field enhancement and produce the low damping mechanism. Second, for a host matrix, we choose the low refractive index $(n)$ to decrease the EM energy outflow into the host matrix. However, two main variables, including geometric factors and operational frequency, are coupled with each other, thus the SP dispersion curves and damping relations can be entirely tailored by the geometry of MNW and EM fields. Thus, a rigorous analysis optimizing the SP guiding performance of MNW is, therefore, not only desirable, but also essential to develop SP devices based on MNW.

In general, some numerical simulation [6] and analytical calculation [7] can be used to optimize the SP propagation along the MNW. For example, the FDTD or finite-element method (FEM) based on the EM field solvers with a longer simulation time validates the SP propagation along the MNW. As an alternative of the EM field solvers, the $R L C$ circuit elements of MNPs have been proposed recently [13], [14]. Moreover, these equivalent-circuit elements are directly incorporated into the circuit simulations, such as HSPICE, thus leading to the possibilities of optimization of SP propagation in the circuit-level simulation. In addition, this equivalent-circuit modeling leads to fast system-level simulation combined with conventional photonic components [15]-[17].

In this paper, we focus on the rigorous way to present the equivalent-circuit elements of the MNW. To establish nonlinear circuit block of SP modes along the MNW, we have used to 
physical approach to define nonlinear equivalent-circuit elements ( $R, L, C$, and $G$ ) are capable of mimicking SP behaviors, such as SP dispersion relation $k(\omega)$, damping curves $\alpha(\omega)$, and power flow $P(\omega)$. To demonstrate the equivalent-circuit elements of SP MNW based on physical approach [18], [19], we need to develop the physical equation formulations. First, we investigate the dispersion relation and damping curve of nonradiative SP waves along the MNW by using Newton-Raphson algorithm. We can gain an intuitive understanding of the low-pass TL model by investigating the SP dispersion relation. For instance, in the conventional TL model, the per-unit-length (PUL) circuit parameters are derived from the integral of Maxwell's equation. However, contrary to the conventional TL approach, our circuit elements are based on the dispersion relation and damping curve because the propagation constant is determined by PUL circuit elements: $\alpha+j k=\sqrt{(r+j \omega l)(g+j \omega c)}$, where $r, l$, $g$, and $c$ denote resistance per unit length, inductance per unit length, conductance per unit length, and capacitance per unit length. Second, to be complete description of all circuit parameters, the current $(I)$ and voltage $(V)$ should be described as the physical quantity. For example, in the transmission-line (TL) analysis, the EM fields of a TEM satisfy a static field distribution in a transverse plane, thus voltage and current are uniquely defined as a transverse plane based on the integration of $\mathrm{E}$ or $\mathrm{H}$ fields. However, because the SP modes generate TM modes, we have used the net power flow concept to obtain effective characteristic impedance $\left(Z_{c}=V / I\right)$ in analogy with the calculation of radiation impedance for an antenna. In this way, we can calculate the effective voltage as a physical meaning. Especially, among many SP propagation modes, we focus on the dominant TM propagation mode $(n=0)$ with no surface charge variation in the $\theta$-direction, thus easily obtaining the effective current and effective voltage along the MNW. Furthermore, the lumped-element model and time-harmonic current (or voltage) distribution lead us to calculate the low-pass transmission parameters such as series impedance $(Z)$ and shunt admittance $(Y)$. Third, the finite-difference (FD) methods such as T-cells, $\pi$-cells, and half-cells enable us to obtain the equivalent-circuit parameters of MNW: resistance $(R)$, capacitance $(C)$, inductance $(L)$, or conductance $(G)$. Fourth, these equivalent-circuit parameters of the MNW are validated by HSPICE and FDTD simulation. Finally, we develop the equivalent-circuit networks for the parallel MNWs.

\section{DisPERSION RELATION AND DAMPING CURVE OF SP IN THE MNW}

In the case of flat metallic surface, the SP dispersion can be calculated by solving the Maxwell equations. In detail, this relationship is given by the following well-known formula [20]:

$$
k_{\mathrm{sp}}=k_{f} \sqrt{\frac{\varepsilon_{s} \varepsilon_{m}}{\varepsilon_{s}+\varepsilon_{m}}}
$$

where $k_{f}=\omega / c$ is the free-space wave vector, $\varepsilon_{s}$ is the permittivity of the surrounding material, and $\varepsilon_{m}$ is the frequency dependent permittivity of the metallic structure. However, in order to calculate the SP dispersion in the MNP, we shall consider the subwavelength cylindrical structures with a cross section of radius $R_{0}\left(R_{0} \ll \lambda\right)$ in the longitudinal $z$-axis, transverse $r$, and $\theta$ plane. In the case of subwavelength circular geometry, we assume that the nonradiative near field energy is transported along the $z$-direction, and the phase constants of $r$ and $\theta$ are imaginary because EM wave satisfies the Heisenberg uncertainty principle $(\Delta x \Delta p>\hbar)$. Furthermore, unlike a conventional optical waveguide, the SP modes are purely transverse magnetic (TM) modes because the surface charge generates a transverse magnetic field at the interface between a metal structure and a surrounding matrix. Therefore, the nonradiative EM fields in the metal core $(r<R)$ are given by as follows [7]:

$$
\begin{aligned}
& E_{z 1}=A I_{n}\left(k_{\rho 1} r\right) \quad E_{r 1}=A \frac{i k_{z}}{k_{\rho 1}} I_{n+1}\left(k_{\rho 1} r\right) \\
& H_{\theta 1}=A \frac{i \omega \varepsilon_{1}}{k_{\rho 1}} I_{n+1}\left(k_{\rho 1} r\right)
\end{aligned}
$$

and EM fields in the host matrix $(r>R)$ are given by

$$
\begin{aligned}
& E_{z 1}=B K_{n}\left(k_{\rho 1} r\right) \quad E_{r 1}=-B \frac{i k_{z}}{k_{\rho 2}} K_{n+1}\left(k_{\rho 2} r\right) \\
& H_{\theta 1}=-B \frac{i \omega \varepsilon_{2}}{k_{\rho 2}} K_{n+1}\left(k_{\rho 2} r\right)
\end{aligned}
$$

where $k_{\rho i}=\left(k_{z}^{2}-\varepsilon_{i} \mu \omega^{2}\right)^{1 / 2}, i=1,2$, are transverse phase components, $I_{n}$ and $K_{n}$ are the $n$ th-order exponential growth and decaying functions for the modified Bessel equation, and $R$ is the radius of MNW. From boundary conditions with tangential EM field components continuity, 1-D SP transcendental equation can be obtained

$$
\frac{K_{n}\left(k_{\rho 2} R\right)}{I_{n}\left(k_{\rho 1} R\right)}=-\frac{\varepsilon_{2} k_{\rho 1} K_{n+1}\left(k_{\rho 2} R\right)}{\varepsilon_{1} k_{\rho 2} I_{n+1}\left(k_{\rho 1} R\right)} .
$$

Newton-Raphson numerical algorithm leads us to calculate the dispersion relation and damping curves in the function of frequency. In this calculation, we have used the complex dielectric constant of $\mathrm{Ag}, \varepsilon_{r}(\omega)=\varepsilon_{r e}(\omega)+j \varepsilon_{i m}(\omega)$, obtained from the cubic spline method by using the Johnson's experimental optical data [21]. Fig. 1(a) shows the SP dispersion relations and damping curves calculated using (4) for SP propagating along the Ag MNW with radius $R=10 \mathrm{~nm}$ that is surrounded by freespace medium $\left(\varepsilon_{2}=1\right)$. These dispersion curves are plotted as a function $k_{z} / k_{0}\left(k_{0}=\omega_{p} / c\right.$, where $\omega_{p}$ is the bulk plasma frequency: $9.183 \times 10^{15} \mathrm{rad} / \mathrm{s}$, where $c$ is the light velocity), which has the nondimensional wave number. As in the inset of Fig. 1(a), the symmetric fundamental mode $(n=0)$ with no surface charge variation in the $\theta$-direction leads to small momentum mismatch between free space and SP wave, and small damping coefficients compared to the higher other modes $(n>0)$. Moreover, as shown in Fig. 1(a), for a low frequency, SP modes asymptotically approaches the light line and small damping loss and, at large $k_{z}$, the SP modes approaching the cutoff frequency $0.707 \omega_{p}$ has the strong damping loss. This behavior is similar to that of flat metallic SP mode. However, compared to zero curvature structure that denotes metallic flat surface, 

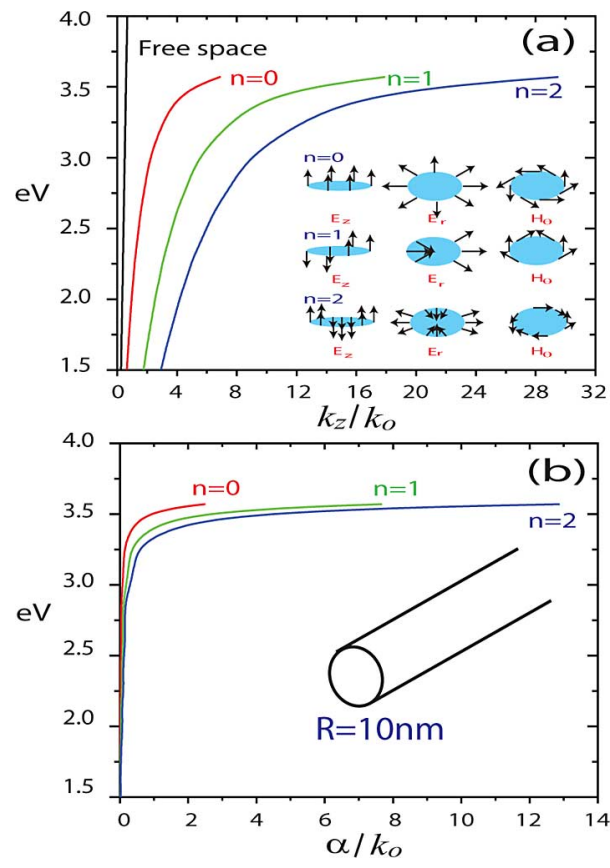

Fig. 1. (a). Dispersion relations of $\mathrm{Ag}$ (silver) $\mathrm{MNW}$ with $\operatorname{radius}(R=10 \mathrm{~nm})$. (b) Damping curves of $\mathrm{Ag}$ (silver) MNW with radius $(R=10 \mathrm{~nm})$. These calculations are obtained from the cubic spine method by using Johnson's experimental optical data $\left(k_{0}=\omega_{p} / c, \omega_{p}\right.$ is the bulk plasma frequency, $c$ is the light velocity). $H$ fields have the following term $H=H_{0} \exp (-\alpha z) \exp (i \omega t-$ $\left.i k_{z} z\right)$.

positive charge distribution and circular electron confinement with small geometry $(R=10 \mathrm{~nm})$ induce the strong momentum. For example, for $\omega=0.4 \omega_{p}$, the momentum of fundamental mode $(n=0)$ numerically found to be $k_{z} \sim 9.4 k_{f}\left(k_{f}=\omega / c\right)$. However, in the case of metallic flat structure with free-space surrounding material $\varepsilon_{2}=1$, for $\omega=0.5 \omega_{p}$, the momentum of SP analytically found to be $k_{\mathrm{sp}} \sim 1.22 k_{f}$ by using (1). This strong momentum mismatch of 1-D SP wave is responsible for the EM coupling problem and multiple scattering interferences between light and SP wave.

In order to check the size contraction of nonradiative SP propagation along the MNW, we have simulated for MNWs with different radius. In Fig. 2(a) and (b), the dispersion relations and damping curves obtained by Newton-Raphson algorithm for the case of $n=0$ (fundamental propagation mode) are also plotted. As can be seen, small radius leads to increase the momentum and attenuation coefficient. As expected, the size contraction has a significant impact on the photonic behavior of SP wave.

\section{NANO-TL MODELING OF MNW}

It has been shown that SP modes along the MNW have the cutoff frequency $\omega_{0}=0.707 \omega_{p}$. Below the cutoff frequency $\left(\omega_{0}\right)$, SP modes can be generated along the MNW. Intuitively, this behavior can be interpreted as a low-pass lumped-TL model with series $L$ (inductance per unit length) and $C$ (capacitance per unit length), having the cutoff frequency $\omega_{0}=2 /(L C)^{1 / 2}$, as shown in Fig. 3. This dispersion analysis of TL model provides a theoretical physical concept for the energy transfer mecha-
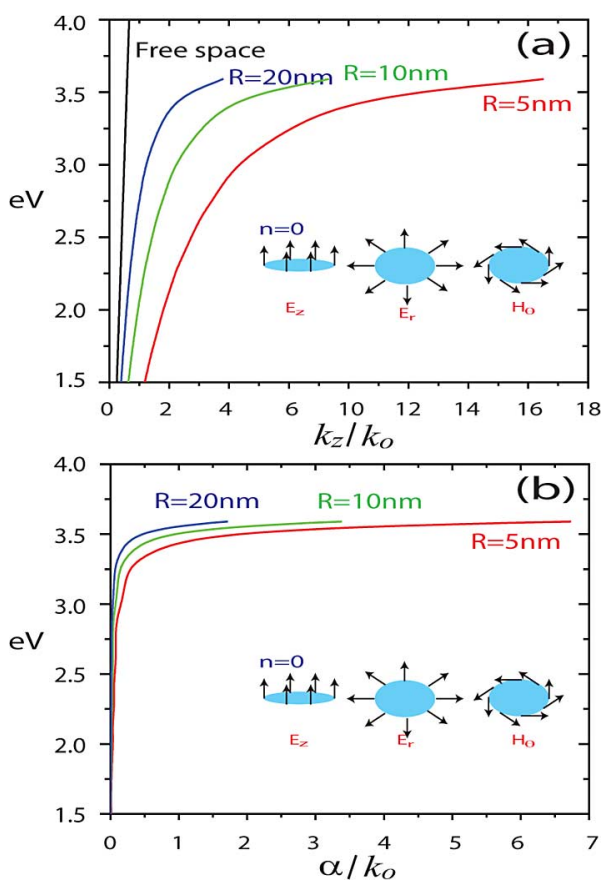

Fig. 2. (a) Dispersion relations of $\mathrm{Ag}$ (silver) MNW with different radii. (b) Damping curves of $\mathrm{Ag}$ (silver) MNW with different radii. These calculations are obtained from the cubic spine method by using Johnson's experimental optical data $\left(k_{0}=\omega_{p} / c, \omega_{p}\right.$ is the bulk plasma frequency, $c$ is the light velocity). $H$ fields have the following term $H=H_{0} \exp (-\alpha z) \exp \left(i \omega t-i k_{z} z\right)$.

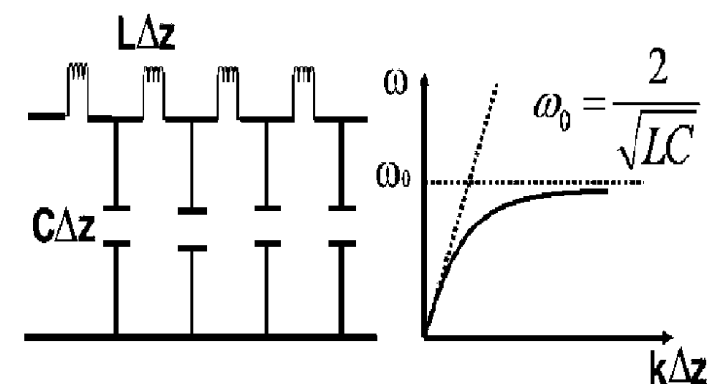

Fig. 3. Lumped low-pass TL model and dispersion curve.

nism along the MNW. In this paper, we examine a simple case of the TL modeling of the MNW. To calculate the TL parameters, we begin to find out the basic physical mechanism in the MNW. The electron cloud oscillation around the metallic surface can be generated by a local field excitation. These oscillatory electron clouds give rise to unique EM field distribution, where the magnetic fields have only axial component $H_{\theta}$, which means the TM waves. If we consider the SP damping factor, SP energy transfer can be modeled as a low-pass lumped-TL consisting of inductance, capacitance, resistance, and conductance, as shown in Fig. 4(c). Furthermore, in order to calculate these circuit parameters, it is necessary to evaluate the current and voltage as a physical quantity. In general, the voltage and current of TEM wave in the usual fashion as integral in the transverse 
(a)

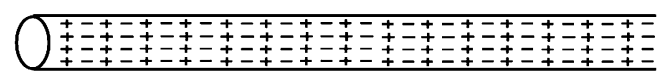

(b)

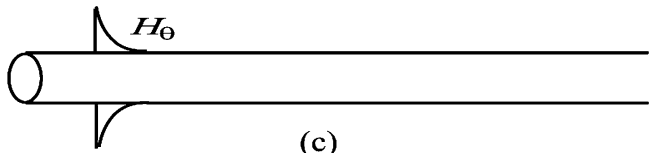

(c)

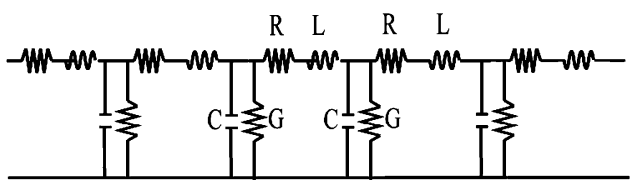

Fig. 4. Equivalent models of nonradiative SP wave along the MNW with fundamental mode $n=0$ with no surface charge variation in the $\theta$-direction. (a) Electron charge oscillation model. (b) EM field distribution. (c) Equivalent lumped-low-pass TL model.

plane:

$$
I(z, t)=-\oint_{c^{\prime}} \vec{H}_{\theta} \cdot d \overrightarrow{l^{\prime}} \text { and } V(z, t)=-\int_{c} \vec{E}_{r} \cdot \overrightarrow{d l} .
$$

For SP wave guiding that cannot support a TEM wave, we cannot define unique voltage and current at a given point along the SP MNW. For these reasons, we choose the equivalent current, equivalent voltage and equivalent impedance for TM lines. Even though they are not unique voltage or current, the equivalent parameters yield the proper physical behavior of the guided wave such as power flow, propagation constant and damping [22]. As a first step, to find out equivalent current flow, we start with the transverse EM field distribution around the MNW. By using the magnetic field continuity at the metallic surface $(\rho=R)$, it is easily found that the EM fields inside the MNW $(\rho<R)$ are given by

$$
\begin{aligned}
& H_{\theta}=H_{\theta}(r=R) \frac{I_{n+1}\left(k_{\rho 1} \rho\right)}{I_{n+1}\left(k_{\rho 1} R\right)} \hat{e}_{\theta} \\
& E_{r}=H_{\theta}(r=R) \frac{k_{z} I_{n+1}\left(k_{\rho 1} \rho\right)}{\omega \varepsilon_{1} I_{n+1}\left(k_{\rho 1} R\right)} \hat{e}_{r} .
\end{aligned}
$$

In the outside region $(\rho>R)$, the EM fields are also given by

$$
\begin{aligned}
& H_{\theta}=H_{\theta}(r=R) \frac{K_{n+1}\left(k_{\rho 2} \rho\right)}{K_{n+1}\left(k_{\rho 2} R\right)} \hat{e}_{\theta} \\
& E_{r}=H_{\theta}(r=R) \frac{k_{z} K_{n+1}\left(k_{\rho 2} \rho\right)}{\omega \varepsilon_{2} K_{n+1}\left(k_{\rho 2} R\right)} \hat{e}_{r} .
\end{aligned}
$$

The current flow is governed by the Ampere law

$$
\nabla \times H=\frac{\partial D}{\partial t}+J_{p}+J_{f}=\frac{\partial\left(\varepsilon_{p} E\right)}{\partial t}+J_{f}
$$

where $J_{p}$ is the polarization current density and $J_{f}$ is the source current density and $\varepsilon_{p}=\varepsilon_{o} \varepsilon_{m}$ represents a free electron model. Moreover, the EM fields originate from the embedded electron charge oscillation of metallic structure, thereby reducing to source-free region with source current $J_{f}=0$. In this paper, we only concentrate on the fundamental $(n=0)$ nonradiative TM mode with no surface charge variation in the $\theta$-direction. With the stokes theorem, the total current flow of fundamental mode $(n=0)$ around the MNW is readily obtained

$$
I_{z}(R)=2 \pi R H_{\theta}(r=R) .
$$

The validity of (11) can be physically explained by oscillating charge distributions inside the MNW $(r \leq R)$, as shown in Fig. 4(a). Since the magnetic field lies in the transverse plane, the induced current around the MNW are directed into the $z$-axis. However, the physical quantity of voltage is not easily obtained by using the integral formulation $E_{r}$, as shown in (5) because the locally distributed oscillating charges around the periphery of the MNW generates not TEM wave, but TM wave with $E_{z}$ field. As an alternative of E-field integration, we have used net power flow over the cross section ( $r-\theta$ plane) to obtain the physical quantity of voltage flow. For example, in a similar way, the radiation impedance for the antenna, such as dipole or linear wire with a current distribution can be obtained by calculating the radiation power flow. This idea is extensible to obtain the effective voltage along the MNW. In detail, the timeaveraged power flow in the $+z$-direction around the MNW at the given point $z$ is given by

$$
\begin{aligned}
P_{\mathrm{net}}^{+} & =P_{z}^{\mathrm{in}}+P_{z}^{\mathrm{out}} \\
& =\frac{1}{2} \int_{A_{\mathrm{in}}} E_{r}^{+} \cdot H_{\theta}^{+*} d S+\frac{1}{2} \int_{A_{\mathrm{out}}} E_{r}^{+} \cdot H_{\theta}^{+*} d S .
\end{aligned}
$$

Then, (12) can be written in terms of current flow

$$
P^{+}=\frac{1}{2} I_{z}^{+}(r=R) I_{z}^{+*}(r=R)\left(Z_{\text {in }}+Z_{\text {out }}\right)
$$

where

$$
Z_{\text {in }}=\frac{k_{z}}{2 \pi R^{2} \omega \varepsilon_{1}} \frac{1}{I_{1}\left(k_{\rho 1} R\right) I_{1}\left(k_{\rho 1} R\right)} \int_{0}^{R} I_{1}\left(k_{\rho 1} \rho\right) I_{1}\left(k_{\rho 1} \rho\right) \rho d \rho
$$

and

$$
\begin{aligned}
Z_{\text {out }}= & \frac{k_{z}}{2 \pi R^{2} \omega \varepsilon_{2}} \frac{1}{K_{1}\left(k_{\rho 2} R\right) K_{1}\left(k_{\rho 2} R\right)} \\
& \times \int_{R}^{\infty} K_{1}\left(k_{\rho 2} \rho\right) K_{1}\left(k_{\rho 2} \rho\right) \rho d \rho .
\end{aligned}
$$

As can be seen, the power flow along the MNW can be classified as the right-handed power flow ( $\left.P_{z}^{\text {out }}\right)$ and left-handed power flow $\left(P_{z}^{\text {in }}\right)$. In detail, outside the MNW, $E_{r}$ and $H_{\theta}$ have right cross product in terms of wave vector $\left(k_{z}\right)$, therefore, $P_{z}^{\text {out }}>0$. However, inside the MNW, $E_{r}$ has reverse direction because of $\varepsilon_{2}<0$, denoting left cross product and denoting $\operatorname{Re}\left(P_{z}^{\mathrm{in}}\right)<0$. The net power flow is the sum of $P_{z}^{\text {out }}$ and $P_{z}^{\text {in }}$. To be specific, we can calculate the $Z_{\text {out }}=201.1$ and $Z_{\text {in }}=-2.4268-0.0709 j$ at $2.0 \mathrm{eV}$ (red spectrum) in the case of MNW with radius $10 \mathrm{~nm}$ based on the SP dispersion relation and damping curve, as shown in Fig. 1 . Because of $Z_{\text {out }} \gg Z_{\text {in }}$, the dominant power flow around the MNW is $P_{z}^{\text {out }}$, thus validating the SP dispersion relation mimicking to the low-pass TL. On the other hand, if the left handed power flow dominates, 
the series impedance $\operatorname{Im}\left(Z \approx j k_{z} Z_{c}\right)$ is negative, thus denoting the high-pass TL to explain the negative refractive EM behavior. Furthermore, this net power flow concept can be used to connect between effective current and effective voltage in the circuit theory. Especially, the complex power flow in the circuit theory can be expressed in terms of complex harmonic voltage and current:

$$
P^{+}=\frac{1}{2} V^{+} I^{+^{*}}=\frac{1}{2} I^{+} Z_{c} I^{+^{*}} .
$$

In this paper, we define the characteristic impedance of the MNW to connect the voltage and current

$$
Z_{c}=Z_{\text {in }}+Z_{\text {out }}
$$

where $Z_{c}=V^{+} / I^{+}$means connecting parameter to relate effective voltage and effective current at a single position. In the case of the MNW, the $Z_{c}$ can be approximately written as

$$
Z_{c} \cong Z_{\text {out }} \text {. }
$$

In a typical TL method, the characteristic impedance $Z_{c}$ provides information about reflection properties. For example, the MNW with $Z_{c}$ is terminated with a load impedance $Z_{L}$, the reflection coefficient can be described by

$$
\Gamma_{L}=\frac{Z_{L}-Z_{c}}{Z_{L}+Z_{c}}
$$

Physically, the terminated load impedance of MNW can be approximately modeled as open circuit load $Z_{L} \rightarrow \infty$ because the current distribution only confined to inside the MNW. However, the MNWs consist of physical lines with various discontinuities and transitions such as gap in MNWs, change in radius, T-junction MNW and coax-to-MNW junction. These discontinuities are required to shunt or series elements across the junction to satisfy the SP physical phenomenon such as EM matching issues. This analysis is much more difficult to analyze and more detail EM analysis is needed to obtain the accurate equivalentcircuit elements. In this paper, we only deal with MNWs without no discontinuities and transitions. Now, it has been shown that the effective voltage and effective current flow at a physical single position are related to net power flows, we now focus on the TL parameters. We have started two fundamental assumptions: the lumped-element model and sinusoidal current (or voltage) waveform. The validity of the lumped-circuit model is that circuit parameters of the MNW are not distributed into a single physical position but are lumped into all length. Thus, the TL equations can be expressed as

$$
\frac{d V}{d z}=-Z I \quad \text { and } \quad \frac{d I}{d z}=-Y V
$$

where $Z$ and $Y$ mean are the distributed series impedance and shunt admittance, respectively. The second assumption denotes that the current (or voltage) in the $+z$-direction can be expressed as follows:

$$
\begin{aligned}
I(z, t) & =I \exp (-\alpha z) \exp \left(i \omega t-i k_{z} z\right) \\
V(z, t) & =V \exp (-\alpha z) \exp \left(i \omega t-i k_{z} z\right)
\end{aligned}
$$
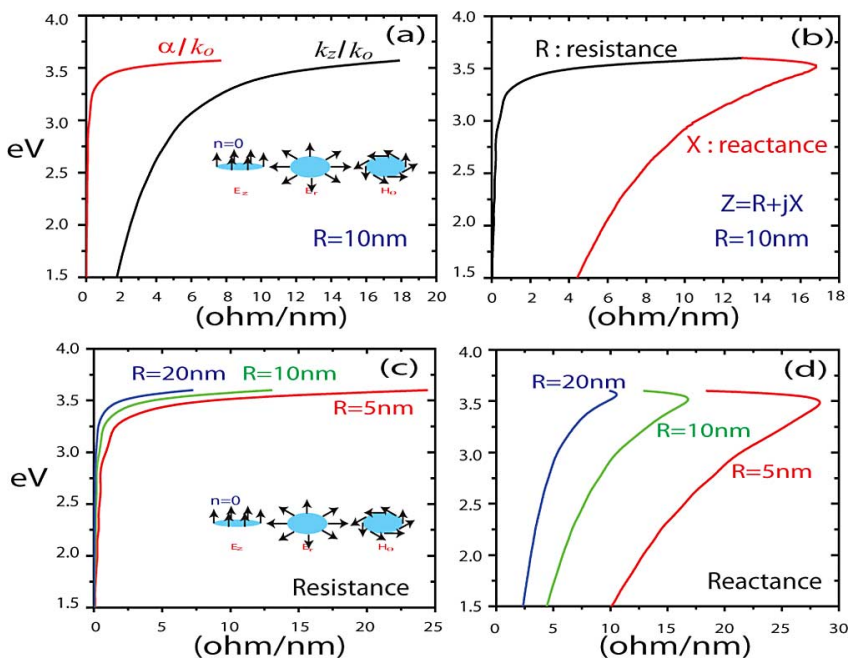

Fig. 5. (a) Plasmon zeroth-mode wave number and damping coefficient of $\mathrm{Ag}$ with radius $10 \mathrm{~nm}$. (b) Impedance $(Z=R+j X)$ of plasmon zeroth-mode of metallic nanowire with radius $10 \mathrm{~nm}$. (c) Resistance of SP zeroth mode as a function of frequency with different radii. (d) Reactance of SP zeroth mode as a function of frequency with different radii.

where $k_{z}$ and $\alpha$ are satisfied with SP transcendental equation. Therefore, with the help of (21), (20) can be described by

$$
\begin{gathered}
\frac{d V(z, t)}{d z}=-\left(\alpha+i k_{z}\right) V(z, t)=-Z I \\
\frac{d I(z, t)}{d z}=-\left(\alpha+i k_{z}\right) I(z, t)=-Y V .
\end{gathered}
$$

With the help of connecting parameter $Z_{c}$ between $V$ and $I$, the lumped series impedance $(Z)$ and shunt admittance $(Y)$ can be expressed as

$$
Z=\left(\alpha+j k_{z}\right) Z_{c} \quad \text { and } \quad Y=\frac{\alpha+j k_{z}}{Z_{c}} .
$$

In the case of sinusoidal excitation, the $Z$ and $Y$ of the lowpass TL can be described as

$$
r+j \omega l=\left(\alpha+j k_{z}\right) Z_{c} \quad \text { and } \quad g+j \omega c=\frac{\alpha+j k_{z}}{Z_{c}}
$$

where $r, l, g$, and $c$ denote resistance per unit length $(R / m)$, inductance per unit length $(L / m)$, conductance per unit length $(\mathrm{G} / \mathrm{m})$, and capacitance per unit length $(\mathrm{C} / \mathrm{m})$, respectively. In the case of the MNW, the damping coefficient $(\alpha)$ can be an order of magnitude of the wave vector $\left(k_{z}\right)$ in the optical spectrum, as shown in Fig. 5(a). Thus, the damping factor $(\alpha)$ should be considered to calculate the TL parameters of the MNW. Fig. 5(b) shows the fundamental mode $(n=0)$ impedance $(Z=$ $R+j X)$ curves for MNW by using the dispersion relation and damping coefficient calculated from the SP dispersion relation. First, positive reactance $(X>0)$ indicates the inductive element because of $Z_{\text {out }} \gg Z_{\text {in }}$, verifying a low-pass lumped TL. As it is expected, the damping coefficient of SP proportionally provides equivalent nanoresistance, as shown in Fig. 5(b). For example, in the blue (optical) spectrum $\left(\omega \sim 4.56 \times 10^{15} \mathrm{rad} / \mathrm{s}\right.$ : 
(a)

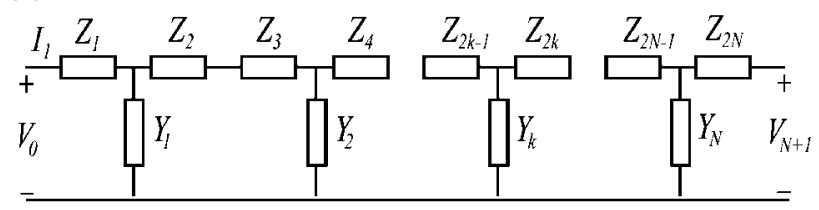

(b) $\quad \mathrm{R} \quad \mathrm{L} \quad \mathrm{R} \quad \mathrm{L}$

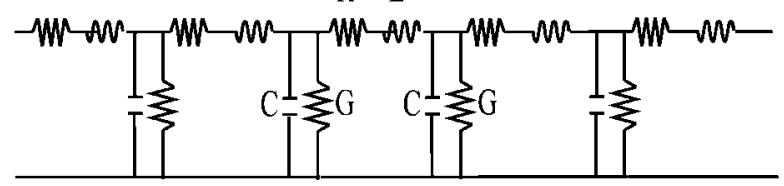

Fig. 6. (a) FD-based element equivalent circuit. (b) T-cell RGLC circuit model of a low-pass TL model.

$3.0 \mathrm{eV}$ ), resistance and reactance of the $\mathrm{MNW}$ with radius $10 \mathrm{~nm}$ approximately have about 0.404 and $10.7 \Omega / \mathrm{nm}$, denoting high damping mechanism. However, in the red (optical) spectrum $\left(\omega \sim 3.04 \times 10^{15} \mathrm{rad} / \mathrm{s}: 2.0 \mathrm{eV}\right)$, resistance and reactance have about 0.101 and $5.82 \Omega / \mathrm{nm}$, denoting small damping mechanism and low impedance compared to blue spectrum. Fig. 5(c) and (d) shows the effect of radius on the impedance of SP propagation along the MNW. As can be seen, the small dimension of the MNW leads to strong attenuation and high reactance. From the curves, as the radius increases twice, the resistance and reactance almost decrease at half values.

\section{EQUivalent-Circuit Modeling FOR SP PROPAGATION ALONG THE MNW}

In previous sections, we have introduced the TL properties of the SP MNW: capacitance per unit length $(\mathrm{C} / \mathrm{m})$, inductance per unit length $(L / m)$, resistance per unit length $(R / m)$, and conductance per unit length $(G / m)$. These parasitic elements have an impact on the photonic behavior of the SP MNW circuit and influence its delay, power attenuation and reliability [23]. Basically, the lossy TL can be analyzed by using two basic approaches: convolution method with impulse response and lumped-element circuit model [24]. In this paper, we focus on the lumped-equivalent-circuit model, thus easily implemented in the existing circuit simulation tools such as Spice simulator [25]-[28]. To demonstrate the lumped-equivalent-circuit model, let the distributed PUL parameters of the MNW be denoted by $Z$ and $Y$, representing series impedance and shunt admittance, respectively. By applying the FD method in (23), the FD discretization with respect to $z$ leads to a RLCG network, as shown in Fig. 6. Among many different FD discretizaion methods such as T-cells, $\pi$-cells, and half-cells, we have simulated the symmetric T-cell method since different cell methods lead to a quite similar result if the cell lengths are small enough. Let us assume that the number of T-cells is $N$, the resistance, inductance, conductance, and capacitance elements of $i$ th T-cell segment can be obtained by

$$
\bar{Z}_{i}=R_{i}+s L_{i}=\frac{Z l}{2 N} \quad \text { and } \quad \bar{Y}_{i}=G_{i}+s C_{i}=\frac{Y l}{N}
$$
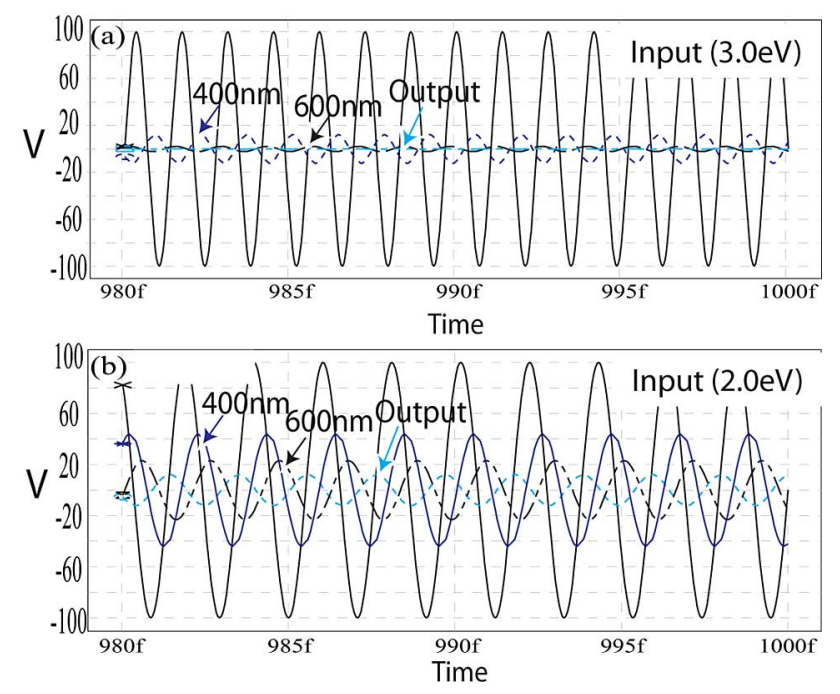

Fig. 7. HSPICE transient analysis of Ag MNW (radius: $10 \mathrm{~nm}$, length: $800 \mathrm{~nm}$ ) obtained by HSPICE simulator: (a) Blue optical frequency $(\omega \sim$ $\left.4.56 \times 10^{15} \mathrm{rad} / \mathrm{s}: 3.0 \mathrm{eV}\right)$. (b) Red optical frequency $\left(\omega \sim 3.04 \times 10^{15} \mathrm{rad} / \mathrm{s}\right.$ : $2.0 \mathrm{eV})$.

where $l$ is the length of the $\mathrm{MNW}$ and $N$ is the number of T-cell segment. If we consider an MNW with a length $l=$ $800 \mathrm{~nm}$, a radius $R=10 \mathrm{~nm}$, and free-space host matrix ( $n=$ 1). By dividing the MNW into 40 sections, T-cell equivalentcircuit parameters at the blue spectrum $\left(\omega \sim 4.56 \times 10^{15} \mathrm{rad} / \mathrm{s}\right.$ : $3.0 \mathrm{eV}$ ) have the values: $R=3.92 \Omega, L=23.25 \mathrm{fH}, G=$ $0.264 \mathrm{mS}$, and $C=1.94 \mathrm{aF}$. On the other hand, at the red frequency $\left(\omega \sim 3.04 \times 10^{15} \mathrm{rad} / \mathrm{s}: 2.0 \mathrm{eV}\right)$, the T-cell equivalentcircuit parameters have the following values: $R=1.01 \Omega, L=$ $19.13 \mathrm{fH}, G=0.0489 \mathrm{mS}$, and $C=0.979 \mathrm{aF}$. In Fig. 7, the transient analysis performed by HSPICE is illustrated as a function of time. In this case, the input source generates a sinusoidal signal with the amplitude $100 \mathrm{~V}$. Four signal waveforms represent the transient voltage at input port, $400 \mathrm{~nm}$ node, $600 \mathrm{~nm}$ node, and output port $(800 \mathrm{~nm})$, respectively. The parasitic elements of blue spectrum $(3.0 \mathrm{eV})$ play a dominant role in SP power attenuation, as shown in Fig. 7(a). As expected in previous sections, to address signal dissipation, it is necessary to decrease the operational signal frequency. As shown in Fig. 7(b), the red spectrum leads to transfer SP nonradiative signal with small damping factors compared to blue spectrum operation.

To confirm the following equivalent-circuit parameters of MNW, we have conducted 3-D scattered FDTD implementation since this approach has some advantages over the total field formulation in that the dispersive calculation error of incident field is negligible and absorbing boundary condition of scattered field can be easily implemented [29]. Based on the frequency dependent FDTD, our simulation volume is chosen as a 3-D rectangular box with dimension $1000 \times 300 \times 300 \mathrm{~nm}^{3}$. We consider an $\mathrm{Ag} \mathrm{MNW}$ with a length of $800 \mathrm{~nm}$ and a radius of $10 \mathrm{~nm}$, which is surrounded by a free space $(n=1)$. For demonstrating a local nonradiative SP propagation along the MNW, the incident plane wave, polarized parallel to the longitudinal axis of the MNW, irradiates at the input terminal of the 


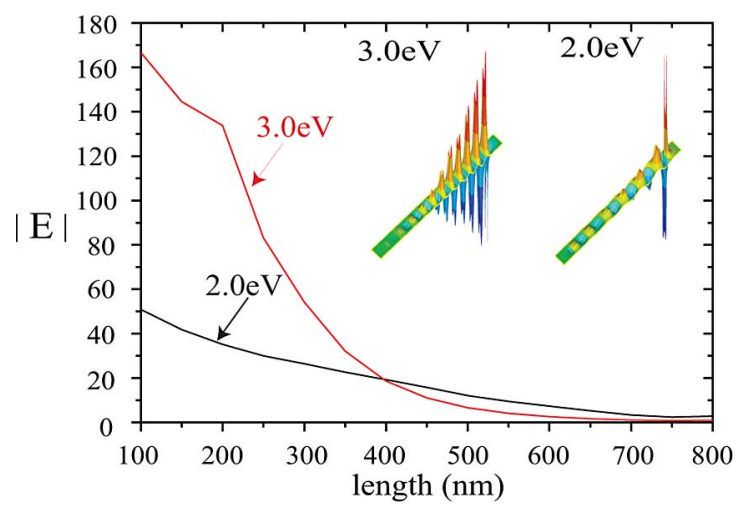

Fig. 8. Average E-field density in the Ag MNW (radius $=10 \mathrm{~nm}$ ) performed by FDTD. These values are calculated at the positions at $15 \mathrm{~nm}$ offset positions ( $z$-directed) from the axis of MNW.
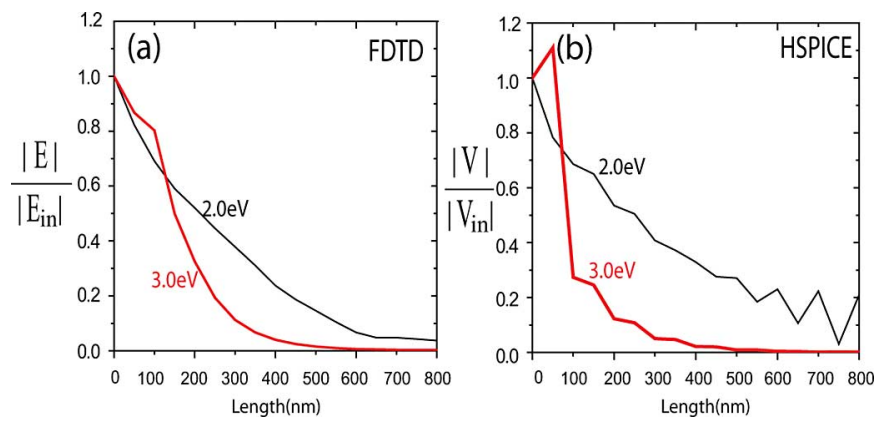

Fig. 9. (a) Average E-field density in the Ag plasmon wire $(r=10 \mathrm{~nm})$ calculated by using FDTD simulation. (b) Average voltage of the Ag plasmon wire (radius $=10 \mathrm{~nm}$ ) calculated by HSPICE simulation based on the circuit parameters. These values are normalized to the input values.

MNW. Accordingly, we obtain the average E-field density at different positions. These values are calculated at the positions at $15 \mathrm{~nm}$ offset positions ( $z$-directed) from the axis of MNW. The inset of Fig. 8 shows $x$-component of the scattered field ( $x-y$ plane) calculated by using the FDTD simulation for two different frequencies. As shown in Fig. 8, even though the blue frequency $\left(\omega \sim 4.56 \times 10^{15} \mathrm{rad} / \mathrm{s}: 3.0 \mathrm{eV}\right)$ generates strong subwavelength localization at the input port of SP MNW because the resonant SP frequency of Ag MNW locates at the blue spectrum, power attenuation become dominant in this spectrum. In contrast, red spectrum $\left(\omega \sim 3.04 \times 10^{15} \mathrm{rad} / \mathrm{s}: 2.0 \mathrm{eV}\right)$ produces weak subwavelength confinement and slowly attenuating near field along the SP MNW. In Fig. 9, the attenuation of E-field (FDTD) and voltage (HSPICE) are compared. These values at different positions are normalized to the input values. The closely agreement between FDTD and HSPICE validates the MNW equivalent-circuit parameters. As the frequency reduces, small damping coefficients lead to multiple reflections between two terminals of MNW. When the propagation speed and operational frequency become sufficiently fast, the multiple reflection plays an important role in the behavior of TL. For example, the Fabry-Perot resonator as the silver MNW with diameter around $100 \mathrm{~nm}$ is experimentally demonstrated [30]. In detail, by using the Fabry-Perot resonator analysis, if the electric amplitude of
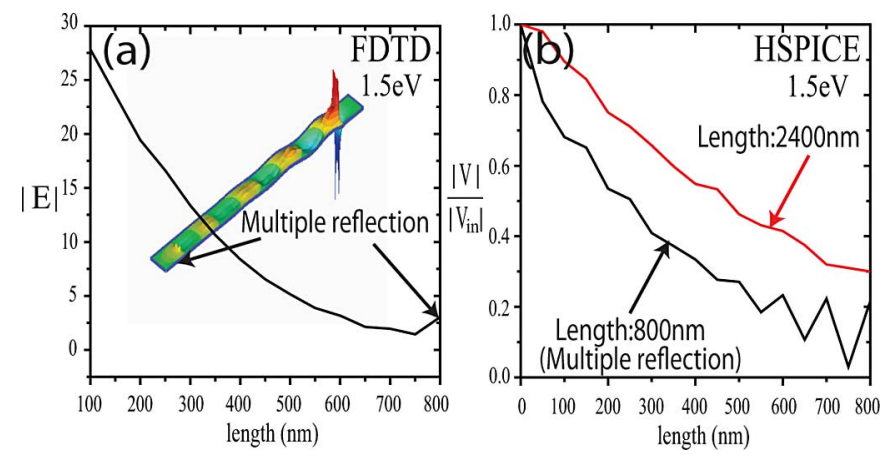

Fig. 10. (a) Average E-field density in the $\mathrm{Ag}$ plasmon wire (radius $=10 \mathrm{~nm}$, length $=800 \mathrm{~nm})$ at a frequency $\left(\omega \sim 2.28 \times 10^{15} \mathrm{rad} / \mathrm{s}: 2.0 \mathrm{eV}\right)$ by FDTD. (b) Average voltage of the $\mathrm{Ag}$ plasmon wire (radius $=10 \mathrm{~nm}$ ) calculated by HSPICE simulation based on the circuit parameters. Black line denotes the $N=40$ segments with length $l=800 \mathrm{~nm}$ and red line indicates the $N=120$ segments with length $l=2400 \mathrm{~nm}$, respectively.

the SP wave after $2(k-1)$ traveling is $A_{k}$, the amplitude gain loss per pass between MNW is $g=\exp (-\alpha L)$ and the phase change between adjacent wave $k$ and $k+1$ is the $\theta=k_{z} L$. The amplitude $A_{k}$ has the following relations: $A_{1}=A_{0} t^{2} g \exp (j \theta)$ and $A_{k+1}=A_{k} r^{2} g^{2} \exp (2 j \theta)$, if $k \geq 1$, where $A_{0}$ denotes the amplitude of incident electric field in the MNW. Thus, the SP transmittance is given by

$$
T_{\text {plasmonic }}=\left|\frac{A_{\text {trans }}}{A_{0}}\right|^{2}=\frac{(1-R)^{2} g^{2}}{\left(1-R g^{2}\right)^{2}+4 R g^{2} \sin ^{2}\left(k_{z} L\right)}
$$

where $R=r^{*} r$ denotes the reflectivity of SP wave in the MNW. As seen from (26), the SP transmittance can be controlled by reflectance coefficient, wave number, and guiding length. To generate the multiple reflections along MNW, we now focus on the SP guiding mechanism in the MNP at a frequency $1.5 \mathrm{eV}$ $\left(\omega \sim 2.28 \times 10^{15} \mathrm{rad} / \mathrm{s}: 1.5 \mathrm{eV}\right)$. In Fig. 10(a), the average E-field density for $\mathrm{Ag} \mathrm{MNW}$ (radius $=10 \mathrm{~nm}$ ) is obtained by FDTD simulation. As can be seen, the extraordinary EM field enhancement at the destination terminal can be explained by the multiple reflections between MNW. In order to check the effect of multiple reflections, we have conducted two different equivalent-circuit models with different T-cell segments. Black line denotes the $N=40$ segments with length $l=800 \mathrm{~nm}$ and red line indicates the $N=120$ segments with length $l=$ $2400 \mathrm{~nm}$, respectively. Both all T-cell equivalent-circuit parameters at the frequency $1.5 \mathrm{eV}$ have the values: $R=0.306 \Omega$, $L=19.39 \mathrm{fH}, G=0.0127 \mathrm{mS}$, and $C=0.749 \mathrm{aF}$. In the case of $N=120$ segment (length $=2400 \mathrm{~nm}$ ), the termination of the line appears an infinite extension termination line because damping effects leads to small portion of reflection wave. Fig. 10(b) gives us a clue on how multiple reflections take an affects on the transient behavior of MNP. As apparent from figure, multiple reflections generate a complex voltage behavior at the destination and rapidly decreasing voltage waveform compared to infinite extension TL. 
(a)
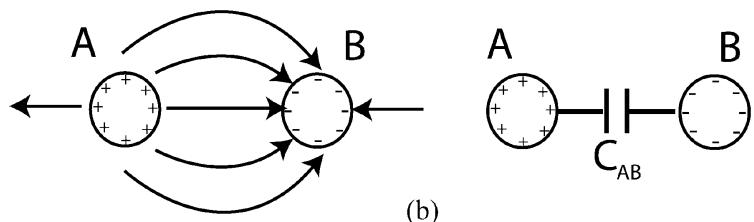

(b)
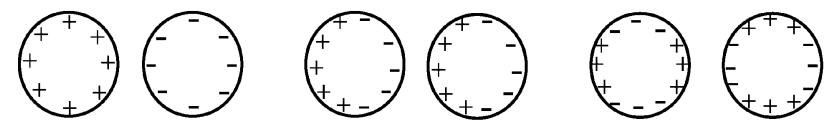

(c)

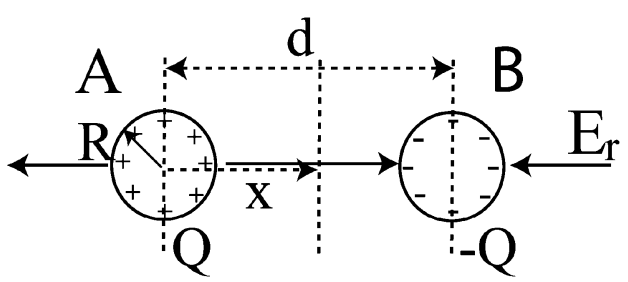

Fig. 11. (a) Electric field distribution and mutual capacitance model. (b) Schematic of monopole, dipole, and quadrupole coupling. (c) Geometry of two identical MNWs

\section{Circuit Network of MNWS}

Until now, we have concentrated on the single MNW structure. However, when MNWs array are close together, power can be coupled between the MNWs array due to the interaction of the EM fields of the MNW lines [31], [32]. When signal pulses are transmitted between MNWs, two fundamental mechanisms are strongly related: 1) electric-field coupling [24], [33], [34], and 2) magnetic-field coupling [35]. In this paper, we concentrate on the electric static coupling because the electrostatic coupling is dominant compared to magnetic interaction [32], [33], [36]-[40]. In detail, the electrostatic coupling is modeled as the mutual capacitance, as shown in Fig. 11(a). In the case of MNWs, the electrostatic coupling is evaluated by using the multiple expansion method. As shown in inset of Fig. 1(a), the charge distribution of the MNW can be expanded as sum of monopole, dipole, quadrupole, etc. Fig. 11(b) shows the schematic depiction of electrostatic coupling of multiple expansions. In the case of nonradiative TM modes, at $n=1$ and $n=2$, dipole and quadrupole electrostatic coupling mimics the electric coupling between MNWs. In the case of fundamental mode $(n=0)$, the monopole electrostatic field distribution allows us to calculate the mutual capacitance $C_{A B}$ between two MNWs. In simplicity, we consider the $n=0 \mathrm{TM}$ propagation mode and corresponding mutual capacitance $C_{A B}$ between two identical wires $(r=R)$ with spatial distance $d$ between their axes, as shown in Fig. 11(c). At the point $x(x \geq R)$, the $E_{r}$ field can be calculated as a result of superposition of the $E_{r}$ fields from $A$ wire and $B$ wire, i.e.,

$$
E_{r 1}=-B \frac{i k_{z}}{k_{\rho 2}}\left[K_{1}\left(k_{\rho 2} x\right)+K_{1}\left(k_{\rho 2}(d-x)\right)\right] .
$$

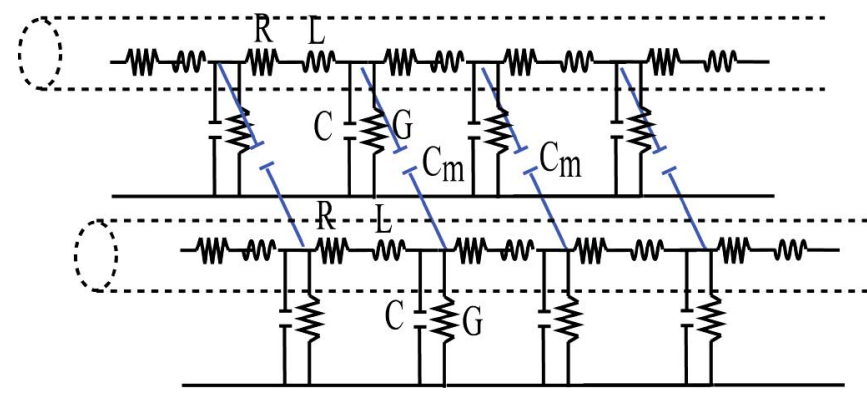

Fig. 12. Circuit model of the parallel MNW wires.

The surface charge $Q$ at $r=R$ is calculated by using the Gauss's law

$$
\frac{Q}{2 \pi R \varepsilon_{0}}=-B \frac{i k_{z}}{k_{\rho 2}} K_{1}\left(k_{\rho 2} R\right)-A \frac{i k_{z}}{k_{\rho 1}} I_{1}\left(k_{\rho 1} R\right) .
$$

From boundary conditions with tangential magnetic-field components continuity, we obtain the following constitutive relation:

$$
\frac{A}{B}=-\frac{k_{\rho 1} \varepsilon_{2} K_{1}\left(k_{\rho 2} R\right)}{k_{\rho 2} \varepsilon_{1} I_{1}\left(k_{\rho 1} R\right)} .
$$

Substituting (29) into (28) yields

$$
\frac{Q}{2 \pi R \varepsilon_{0}}=-B \frac{i k_{z}}{k_{\rho 2}}\left[K_{1}\left(k_{\rho 2} R\right)-\frac{\varepsilon_{2}}{\varepsilon_{1}} K_{1}\left(k_{\rho 2} R\right)\right] .
$$

For example, in the case of Ag NMW surrounded by a free space, the $\varepsilon_{0} / \varepsilon_{\mathrm{Ag}}\left(=\varepsilon_{2} / \varepsilon_{1}\right)$ term goes to zero at the low operational frequency, thus demonstrating the perfect conductor model of a metal at the low frequency. The voltage between two MNWs $(d \gg R)$ can be approximately obtained by the integration of $E_{r}$

$$
V \approx \int_{R}^{d-R} E_{r} d x
$$

We obtain the mutual capacitance $\left(C_{A B}=Q / V\right)$ between two MNWs

$$
C_{A B} \approx \frac{\operatorname{Re}\left[\pi R \varepsilon_{0} K_{1}\left(k_{\rho 2} R\right)\left(1-\left(\varepsilon_{2} / \varepsilon_{1}\right)\right)\right]}{\left[\int_{R}^{d-R} K_{1}\left(k_{\rho 2} x\right) d x\right]} .
$$

Let us assume the two $\mathrm{Ag}$ MNWs with radius $R=20 \mathrm{~nm}$, spatial distance $d=60 \mathrm{~nm}$ and free-space host matrix $(n=1)$. Equation (32) shows that the mutual capacitance of $\mathrm{Ag}$ wire has the value $C_{A B}=0.1240 \mathrm{nF} / \mathrm{m}$ (at $3.0 \mathrm{eV}$ ) and $C_{A B}=$ $34.47 \mathrm{pF} / \mathrm{m}$ (at $2.0 \mathrm{eV}$ ), respectively. To be specific, we construct the equivalent-circuit networks for a coupled MNWs line, as shown in Fig. 12. For example, if we consider two identical MNWs with a length $l=800 \mathrm{~nm}$ and divide the MNWs into 40 sections, T-cell equivalent-circuit parameters at $2.0 \mathrm{eV}$ have the following values: $R=1.01 \Omega, L=19.13 \mathrm{fH}, G=0.0489 \mathrm{mS}, C$ $=0.979 \mathrm{aF}$, and $C_{m}=0.6893 \mathrm{aF}$. This coupled circuit network can be analyzed by using the even- and odd-mode analysis [41] or HSPICE circuit simulator [27], [42]. Furthermore, this analysis based on the electrostatic coupling can be extended to evaluate the crosstalk between multiple MNWs. 


\section{CONCLUSION}

We have studied the equivalent-circuit modeling of SP propagation along the MNW. Our proposed equivalent-circuit model provides a basic understanding of the nature of SP propagation along the MNW and distributed circuit elements, and of how operational frequency and guiding geometry affect the performance of MNW circuitry. Even though SP of MNW in the near-infrared and visible spectrum has severe limitations as a longer signal interconnect, the fast velocity and high operational frequency enabled by SP wave will provide a novel approach to obtain ultrafast speed integrated circuitry. Furthermore, this equivalent-circuit modeling will aid us in the further analysis and optimization of complex SP devices.

\section{ACKNOWLEDGMENT}

The authors would like to thank the anonymous reviewers for their helpful suggestions and comments. The authors would also like to thank Dr. G. Pomrenke of Air Force Office of Scientific Research for encouraging them to develop the theoretical modeling of the signal propagation through chain of nanoparticles (MNP's) and nanowire (MNW).

\section{REFERENCES}

[1] C. Gunn, "CMOS photonics for high-speed interconnects," IEEE Micro, vol. 26, no. 2, pp. 58-66, Mar--Apr. 2006.

[2] W. H. Wu, L. A. Bergman, A. R. Johnston, C. C. Guest, S. C. Esener, P. K. L. Yu, M. R. Feldman, and S. H. Lee, "Implementation of optical interconnections for VLSI," IEEE Trans. Electron Devices, vol. ED-34, no. 3, pp. 706-714, Mar. 1987.

[3] M. R. Feldman, S. C. Esener, C. C. Guest, and S. H. Lee, "Comparison between optical and electrical interconnects based on power and speed considerations," Appl. Opt., vol. 27, pp. 1742-1751, 1988.

[4] S. A. Maier, P. G. Kik, H. A. Atwater, S. Meltzer, E. Harel, B. E. Koel, and A. A. G. Requicha, "Local detection of electromagnetic energy transport below the diffraction limit in metal nanoparticle plasmon waveguides," Nature Mater, vol. 2, pp. 229-232, Apr. 2003.

[5] S. A. Maier, P. G. Kik, and H. A. Atwater, "Optical pulse propagation in metal nanoparticle chain waveguides," Phys. Rev. B, vol. 67, pp. 2054021-205402-5, May 15, 2003.

[6] J. C. Weeber, A. Dereux, C. Girard, J. R. Krenn, and J. P. Goudonnet, "Plasmon polaritons of metallic nanowires for controlling submicron propagation of light," Phys. Rev. B, vol. 60, pp. 9061-9068, Sep. 1999.

[7] J. Takahara, S. Yamagishi, H. Taki, A. Morimoto, and T. Kobayashi, "Guiding of a one-dimensional optical beam with nanometer diameter," Opt. Lett., vol. 22, pp. 475-477, Apr. 1, 1997.

[8] S. A. Maier, M. L. Brongersma, and H. A. Atwater, "Electromagnetic energy transport along Yagi arrays," Mater. Sci. Eng. C-Biomimetic Supramol. Syst., vol. 19, pp. 291-294, Jan. 2, 2002.

[9] M. Quinten, A. Leitner, J. R. Krenn, and F. R. Aussenegg, "Electromagnetic energy transport via linear chains of silver nanoparticles," Opt. Lett., vol. 23, pp. 1331-1333, Sep. 1, 1998.

[10] J. J. Greffet, "Nanoantennas for light emission," Science, vol. 308, pp. 1561-1563, Jun. 2005.

[11] A. Ono, J. Kato, and S. Kawata, "Subwavelength optical imaging through a metallic nanorod array," Phys. Rev. Lett., vol. 95, pp. 267407-1-2674074, Dec. 2005.

[12] D. E. Chang, A. S. Sorensen, P. R. Hemmer, and M. D. Lukin, "Quantum optics with surface plasmons," Phys. Rev. Lett., vol. 97, pp. 053002-1053002-4, Aug. 2006.

[13] N. Engheta, A. Salandrino, and A. Alu, "Circuit elements at optical frequencies: Nanoinductors, nanocapacitors, and nanoresistors," Phys. Rev. Lett., vol. 95, pp. 095504-1-095504-4, Aug. 2005.

[14] M. Alam and Y. Massoud, "RLC ladder model for scattering in single metallic nanoparticles," IEEE Trans. Nanotechnol., vol. 5, no. 5, pp. 491498, Sep. 2006
[15] A. Deutsch, "Electrical characteristics of interconnections for highperformance systems," Proc. IEEE, vol. 86, no. 2, pp. 315-357, Feb. 1998.

[16] A. R. Djordjevic, T. K. Sarkar, and R. F. Harrington, "Time-domain response of multiconductor transmission lines," Proc. IEEE, vol. 75, no. 6 , pp. 743-764, Jun. 1987.

[17] L. Tzu-Mu and C. A. Mead, "Signal delay in general RC networks," IEEE Trans. Comput.-Aided Des. Integr. Circuits Syst., vol. CAD-3, no. 4 pp. 331-349, Oct. 1984.

[18] L. O. Chua, "Nonlinear circuit foundations for nanodevices, part I: The four-element torus," Proc. IEEE, vol. 91, no. 11, pp. 1830-1859, Nov. 2003.

[19] E. S. Kuh and I. N. Hajj, "Nonlinear circuit theory: Resistive networks," Proc. IEEE, vol. 59, no. 3, pp. 340-355, Mar. 1971.

[20] W. L. Barnes, A. Dereux, and T. W. Ebbesen, "Surface plasmon subwavelength optics," Nature, vol. 424, pp. 824-830, Aug. 14, 2003.

[21] P. B. Johnson and R. W. Christy, "Optical-constants of noble-metals," Phys. Rev. B, vol. 6, pp. 4370-4379, 1972.

[22] D. M. Pozar, Mircowave Engineering. New York: Wiley, 2005.

[23] H. B. Bakoglu and J. D. Meindl, "Optimal interconnection circuits for VLSI," IEEE Trans. Electron Devices, vol. 32, no. 5, pp. 903-909, May 1985 .

[24] T. Dhaene and D. Dezutter, "Selection of lumped element models for coupled lossy transmission-lines," IEEE Trans. Comput.-Aided Des. Integr. Circuits Syst., vol. 11, no. 7, pp. 805-815, Jul. 1992.

[25] Z. Bing, S. L. Dvorak, and J. L. Prince, "Transient simulation of lossy interconnects based on a dispersive hybrid phase-pole macromodel," IEEE Trans. Adv. Packag., vol. 30, no. 2, pp. 321-334, May 2007.

[26] C. R. Paul, "Useful matrix chain parameter identities for the analysis of multiconductor transmission lines (short papers)," IEEE Trans. Microw. Theory Tech., vol. MTT-23, no. 9, pp. 756-760, Sep. 1975.

[27] F. H. Branin, Jr., "Transient analysis of lossless transmission lines," Proc. IEEE, vol. 55, no. 11, pp. 2012-2013, Nov. 1967.

[28] J. Rubinstein, P. Penfield, and M. A. Horowitz, "Signal delay in RC tree networks," IEEE Trans. Comput.-Aided Des. Integr. Circuits Syst., vol. CAD-2, no. 3, pp. 202-211, Jul. 1983.

[29] W. A. Challener, I. K. Sendur, and C. Peng, "Scattered field formulation of finite difference time domain for a focused light beam in dense media with lossy materials," Opt. Exp., vol. 11, pp. 3160-3170, Nov. 2003.

[30] H. Ditlbacher, A. Hohenau, D. Wagner, U. Kreibig, M. Rogers, F. Hofer, F. R. Aussenegg, and J. R. Krenn, "Silver nanowires as surface plasmon resonators," Phys. Rev. Lett., vol. 95, pp. 257403-1-257403-4, 2005.

[31] T. Sakurai, "Closed-form expressions for interconnection delay, coupling, and crosstalk in VLSIs," IEEE Trans. Electron Devices, vol. 40, no. 1, pp. 118-124, Jan. 1993

[32] A. E. Ruehli and P. A. Brennan, "Efficient capacitance calculations for three-dimensional multiconductor systems," IEEE Trans. Microw. Theory Tech., vol. MTT-21, no. 2, pp. 76-82, Feb. 1973.

[33] N. D. Arora, K. V. Raol, R. Schumann, and L. M. Richardson, "Modeling and extraction of interconnect capacitances for multilayer VLSI circuits," IEEE Trans. Comput.-Aided Des. Integr. Circuits Syst., vol. 15, no. 1, pp. 58-67, Jan. 1996.

[34] M. W. Beattie and L. T. Pileggi, "On-chip induction modeling: Basics and advanced methods," IEEE Trans. Very Large Scale Integr. (VLSI) Syst., vol. 10, no. 6, pp. 712-729, Dec. 2002.

[35] G. Zhong and C. K. Koh, "Exact closed form formula for partial mutual inductances of on-chip interconnects," in Proc. 2002 IEEE Int. Conf. Comput. Des.: VLSI Comput. Processors, pp. 428-433.

[36] J. A. B. Faria, "On the partial-capacitance scheme for multiple conductor systems," Microw. Opt. Technol. Lett., vol. 47, pp. 346-349, 2005.

[37] W. Shyh-Chyi, L. Gwo-Yann, and M. Dye-Jyun, "Modeling of interconnect capacitance, delay, and crosstalk in VLSI," IEEE Trans. Semicond. Manuf., vol. 13, no. 1, pp. 108-111, Feb. 2000.

[38] T. Sakurai and K. Tamaru, "Simple formulas for two- and threedimensional capacitances," IEEE Trans. Electron Devices, vol. ED-30, no. 2, pp. 183-185, Feb. 1983.

[39] U. Choudhury and A. Sangiovanni-Vincentelli, "Automatic generation of analytical models for interconnect capacitances," IEEE Trans. Comput.Aided Des. Integr. Circuits Syst., vol. 14, no. 4, pp. 470-480, Apr. 1995.

[40] J. Cong, "An interconnect-centric design flow for nanometer technologies," in Proc. VLSI Technol., Syst., Appl., 1999. Int. Symp., pp. 54-57.

[41] J. Reed and G. J. Wheeler, "A method of analysis of symmetrical fourport networks," IRE Trans. Microw. Theory Tech., vol. 4, pp. 246-252, 1956. 
[42] S. Lin and E. S. Kuh, "Transient simulation of lossy interconnects based on the recursive convolution formulation," IEEE Trans. Circuits Syst. I: Fundam. Theory Appl., vol. 39, no. 11, pp. 879-892, Nov. 1992.

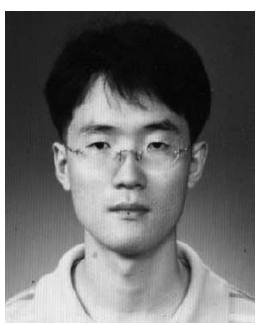

Kyungjun Song received the B.S. degree in mechanical engineering from Seoul National University, Seoul, Korea, and the M.S. degree in mechanical engineering from the University of Michigan, Ann Arbor, in 2002 and 2004, respectively. He is currently working toward the Ph.D. degree in mechanical engineering from the University of Michigan.

$\mathrm{He}$ is a Graduate Student Research Assistant in the Electrical Engineering and Computer Science Department, University of Michigan. His current research interests include the modeling, simulation, and design of plasmonic nanoarchitecture.

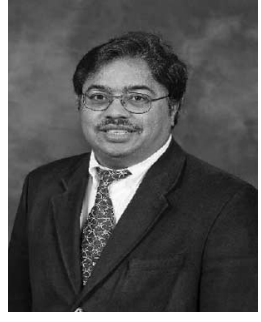

Pinaki Mazumder (F'99) received the Ph.D. degree from the University of Illinois at Urbana-Champaign, Urbana-Champaign, in 1988.

$\mathrm{He}$ is currently a Professor in the Department of Electrical Engineering and Computer Science, University of Michigan (UM), Ann Arbor. He is on leave for 1 year from the UM to serve as the lead Program Director of the Emerging Models and Technologies Program at the U.S. National Science Foundation, Arlington, VA. For six years, he was with industrial Research and Development centers that included AT\&T Bell Laboratories, Murray Hill, NJ, where in 1985, he started the CONES Project - the first C modeling-based very large scale integration (VLSI) synthesis tool at India's premier electronics company, Bharat Electronics Ltd. Bangalore, where he had developed several high-speed and high-voltage analog integrated circuits intended for consumer electronics products. He is the author or coauthor of more than 200 technical papers and four books on various aspects of VLSI research works. His current research interests include current problems in nanoscale CMOS VLSI design, computer-aided design (CAD) tools, and circuit designs for emerging technologies including quantum MOS and resonant tunneling devices, semiconductor memory systems, and physical synthesis of VLSI chips.

Dr. Mazumder is a Fellow of American Association for the Advancement of Science during 2008. He was a recipient of the Digital's Incentives for Excellence Award, BF Goodrich National Collegiate Invention Award, and Defense Advanced Research Projects Agency Research Excellence Award. 